\title{
Les procédés
}

\section{logico-argumentatifs \\ du discours propagandiste dans \\ O Homem e o Cavalo \\ d'Oswald de Andrade}

\author{
Heliane Kohler-Rodrigues \\ Faculté des Lettres et Sciences Humaines \\ de l'Université de Franche-Comté (Besançon)
}

\section{Abstract}

Na peça de Oswald de Andrade publicada em 1934, O Homem e o Cavalo, dois quadros são dedicados à propaganda revolucionária, tendo como contex to enunciativo o mundo socialista, e como agentes discursivos diferentes personagens, representantes do povo soviético.

O objetivo deste trabalho é revelar o mecanismo argumentativo do discurso de propaganda, ou seja, analisar a argumentação propagandista em função dos diferentes argumentos (provas) fornecidos pelos locutores-oradores soviéticos, e explicitar as diferentes operações/construções de "mise en sens" do discurso. A estruturação dos argumentos (antes vs após a Revoluçãa de 1917) revela a presença de 3 tipos de procedimentos lógicoargumentativos: oposição, refutação, ilustração. 


\section{Les procédés logico-argumentatifs du discours propagandiste dans} O Homem e o Cavalo d'Oswald de Andrade

\section{CONSIDÉRATIONS PRÉLIMINAIRES}

$\mathrm{D}$ ans la pièce $O$ Homem e $o$ Cavalo, publiée par Oswald de Andrade en 1934 et représentée pour la première fois en 1990, deux des ses neuf tableaux sont dédiés, dans leur intégralité, à la propagande révolutionnaire ": le tableau VI, intitulé, "A industrialização"et le tableau VII, "A verdade na boca das crianças" - référence au proverbe français, "la vérité sort de la bouche des enfants". Aussi le discours de propagande est-il tenu par deux types de locuteurs-personnages représentant le peuple soviétique qui s'expriment soit de façon médiate, soit anonymement. Dans le premier cas, il s'agit de voix-off (locutions radiophoniques) représentant des personnages de notoriété publique, tant sur le plan politique ( $\mathrm{A} \mathrm{Voz}$ de Stalin), que sur le plan artistique ( A Voz de Eisenstein). Dans le deuxième cas, il s'agit de personnages codés socialement, définis par les traits distinctifs adulte/enfant: $\mathrm{O}$ Médico et Três Crianças Soviéticas.

Dans $O$ Homem e o Cavalo, le discours de propagande communiste est, non seulement de type monologal (allocutions oratoires), mais aussi dialogal, c'est-à-dire intégré dans un réseau interactionnel constitué d'échanges verbaux entre les différents personnages propagandistes et conservateurs. L'adresse au public-spectateur se fait donc par le biais du jeu conversationnel centré autour des agents actoriels. Alors que le tableau VI "met en scène"les discours des locuteurs-orateurs absents scéniquement (voix-off), le tableau suivant exhibe, outre les discours des personnages en interaction verbale, celui d'un personnage propagandiste, O Médico, qui, à la fin du tableau, et à la demande des personnages conservateurs (São Pedro, Mme Icar e Icar), expose longuement les changements sociaux intervenus en Russie après la Révolution d'octobre. Son discours rejoint, par sa structuration 
monologale, ceux des orateurs intervenant dans le tableau précédent. Or, une précision s'impose: le discours monologal n'exclut absolument pas le caractère dialogique des interventions propagandistes. Le discours argumentatif des orateurs-propagandistes répond, en fait, à un contre-discours, c'est-à-dire à des "voix"accusatrices dissemblables, et les marques de réfutation présentes dans le texte témoignent de la teneur dialogique de ce type de discours. Encore faut-il rappeler que selon M.Bakhtine, tout énoncé est foncièrement dialogique dans la mesure où il ne saurait être appréhendé que dans son orientation vers l'auditeur.

Alors que le discours d'agitation de la pièce se déroule dans le contexte capitaliste (tableaux IV et V), le discours de propagande communiste a pour lieu d'énonciation "le monde soviétique", à partir duquel les différentes interventions sont produites; "A cena representa a entrada da maior usina do mundo socialista."2 Axé sur la dialectique présent vs passé, le discours propagandiste révolutionnaire de la pièce repose dans son intégralité sur les catégories axiologiques nouveau (les Soviétiques) vs vieux (les capitalistes) constituant les deux isotopies textuelles dominantes. Tant au niveau sémantique, qu'au niveau syntaxique (emploi récurrent et concomitant du présent et de l'imparfait de l'indicatif; d'énoncés affirmatifs et négatifs), le discours de propagande porte les marques du système axiologique qui le sous-tend et l'organise, en affichant côte à côte le valorisé du non-valorisé. Tout en condamnant l'ordre "ancien", le discours propagandiste s'adresse aux travailleurs pour les inviter à construire, comme en URSS, un monde nouveau, conforme aux exigences du socialisme révolutionnaire.

Tandis que le discours d'agitation met en oeuvre une argumentation pratique, le discours de propagande a pour ancrage thématique le modèle soviétique, la doctrine marxiste: les faits (réalisations économiques et sociales accomplies en URSS) et les jugements forment l'essentiel de l'argumentation révolutionnaire. Axé sur le développement socio-économique survenu en URSS, le discours propagandiste monologal (qu'il soit médiatique ou immédiat) s'articule autour du développement industriel, agricole et social.

Ceci dit, l'analyse de l'argumentation du discours de propagande sera construite à partir du schéma logique sous-jacent au raisonnement propagandiste; ce qui nous permettra, une fois ébauché l'enchaînement conceptuel de l'argumentation révolutionnaire, non seulement de regrouper les différents arguments/preuves exploités par les trois orateurs et d'expliciter les invariants discursifs des respectives allocutions, mais aussi, et surtout, de mettre à jour les différentes opérations/constructions de "mise en sens"du discours, c'est-à-dire de montrer comment l'argumentation investit le discours. 


\section{LA LOGIQUE DU DISCOURS}

C'est à partir d'une premisse catégorique non explicitée textuellement (car considérée comme une affirmation évidente, supposée être admise par l'auditoire - "travailleurs du monde entier") que toute une série d'arguments explicites, fonctionnant en tant que preuves, sont exploités par les orateurs en vue de défendre la conclusion (thèse évaluative) explicite réitérée à maintes reprises dans les interventions des orateurs:

- PREMISSE - (Le développement en URSS est dû à l'implantation du système socialiste)

- ARGUMENTS - (Preuves) - (avant vs après la Révolution d'octobre)

- CONCLUSION - (La construction socialiste représente le développement socio-économique, la modernité)

Ce schéma formel illustre en effet la logique du discours propagandiste, discours axé, rappelons-le, sur les couplages axiologiques passé vs présent, ancien vs moderne, où le "nouveau"(tirant sa force persuasive de son acceptabilité universelle) constitue l'attribut principal de l'objet de l'argumentation: le régime soviétique. En exploitant abusivement des arguments disjoints sémantiquement qui se caractérisent par une opposition "privative" 3 , le discours propagandiste vise à défendre l'axiome idéologique - la thèse du succès de la construction socialiste en URSS construit autour de l'association sémantique valorisante: socialisme = modernité, progrès, amélioration, sous-tendant, par sa propre teneur polémique, le pôle opposé: capitalisme = passé, stagnation, aggravation .

Un examen attentif de la mise en oeuvre des arguments (désignés par une "mise en rapport"de type antériorité vs postériorité) effectuée par le sujet-scripteur dans le discours des orateurs propagandistes, signale la présence de trois types de procédures logico-argumentatives: opposition, réfutation, illustration relevant d'opérations cognitives et langagières constitutives de toute activité langagière. Si les opérations langagières fondent l'agencement des énoncés, ces mêmes opérations ne peuvent être séparables des opérations cognitives motivant cet agencement. Aussi le dire ne peutil être dissociable de l'opérer. En d'autres termes, ce qui est dit dans l'énoncé nous informe de la façon dont ce dire a été construit; ce qui est pensé dans l'énoncé, nous renseigne, en même temps, sur les opérations qui ont été nécessaires pour assurer cette pensée. Étant donné que chaque type 
d'opérations langagières correspond à des configurations différentes d'unités linguistiques, il est nécessaire d'examiner les unités spécifiques qui constituent la trace (i. e. les marqueurs) de ces opérations, liée à l'organisation argumentative du discours.

\section{OPPOSITION}

Reposant sur le "lieu des contraires"4, le discours de propagande exploite donc des arguments dichotomiques qui se manifestent par une structure relationnelle aussi bien au niveau sémantique, qu'au niveau syntaxique. Aussi l'assertivité du discours est-elle sans cesse renforcée par des répétitions et des parallélismes syntaxiques affichant la relation d'opposition entre deux catégories d'arguments juxtaposés, et illustrant les opérations constitutives des propositions, objectivées par le mouvement pendulaire de NEGATION/AFFIRMATION., (AFFIRMATION) / NEGATION spécifique à la "démonstration"qui a trait à la disjonction avant vs après (1917), autrement dit, capitalisme vs socialisme. La disqualification de l'objet pris pour cible se manifeste dans le discours de deux façons: 1) - on nie l'existence d'un certain état de fait (positif) dans le passé, en l'affirmant explicitement au présent; 2) - on nie textuellement l'existence d'un certain état de fait (négatif) au présent, en affirmant implicitement sa présence dans le passé.

Considérons la séquence suivante correspondant au premier type d'opposition - "NEG. imperfeito / AF. presente":

1) - A Voz de Stalin - "Não tínhamos indústria siderúrgica, agora temos! Não tínhamos indústria mecânica, agora temos. Não tínhamos indústria de tratores, agora temos! Não tínhamos indústria de automóveis, agora temos! Não tínhamos indústria química, agora temos! Não tínhamos máquinas agrícolas, agora temos! Não tínhamos liberdade, agora temos!" (p. 188).

$\mathrm{Si}$, d'une part, les répétitions syntagmatiques, les énumérations paradigmatiques, les anaphores, les allitérations - traits spécifiques de la litanie - visent à provoquer chez l'auditeur un effet mnémonique (procédé pédagogique), d'autre part, l'utilisation de ces figures rhétoriques a pour but d'intensifier les oppositions sémantiques (( avant) vs maintenant) et d'accentuer leurs disjonctions axiologiques (dévalorisé (-) vs valorisé $(+)$ ). Malgré la monotonie rythmique due à l'emploi réducteur d'une seule et unique structure phrastique (structure syntaxique et sémantique communes), 
le crescendo du discours s'explique par l'introduction, en position finale, du lexème "liberté"dans la case de l'objet, amplifiant la valeur argumentative de la séquence en question. Aussi les arguments sont-ils présentés dans un ordre discursif qui, selon le sujet-producteur, par le biais de l'orateur, leur donne le plus d'efficacité: c'est-à-dire des plus faibles (faits événementiels - créations d'industries) au plus fort (valeur fondamentale - "a liberdade").

Les marques de l'opposition axiologique, ou plus exactement, les marques du dévalorisé dans l'allocution propagandiste sont donc affichées par l'assertion négative autour de l'axe temporel (passé vs présent) exprimé par le temps verbal (imperfeito do indicativo) et la modalité (negação "não + predicado"). Si l'on examine les énoncés négatifs de la séquence citée ci-dessus, on constate qu'il s'agit de négations "descriptives"s étant donné qu'elles servent à représenter un état de choses antérieur (le retard économique de la Russie). Les énoncés négatifs ("Não tínhamos X") peuvent en fait être paraphrasés par des énoncés positifs, comme, par exemple, "tínhamos um atraso considerável". Aussi les énoncés négatifs ont-ils une force illocutoire d'assertion et un contenu propositionnel négatif. Cette relation peut être schématisée de la façon suivante: $-(\sim \mathrm{p})^{6}$. Tout en étant utilisés pour décrire un certain état de choses, les énoncés négatifs ("não tínhamos X") pourraient néanmoins servir à s'opposer à un énonciateur qui affirmerait le point de vue selon lequel "eles tinham X".

$\mathrm{Si}$, par ailleurs, on essaie de relier les deux énoncés indépendants: "não tínhamos X, agora temos", c'est bien le connecteur argumentatif "mas"qui assure logiquement leur liaison: "Não tínhamos, (mas) agora temos". Or, la description classique du connecteur "mas ("mais")"formulée par Ducrot et Anscombre ${ }^{7}$ stipule que dans les énoncés de type "p mais q", p est présenté comme un argument pour une certaine conclusion implicite (r) et q pour la conclusion inverse, (non-r). $Q$ ayant une force argumentative plus grande que $p$, l'ensemble "p mais q"va dans le sens de non-r. En d'autres termes, les arguments introduits par "mais"sont anti-orientés ${ }^{8}$, c'est-à-dire qu'ils sont présentés comme destinés à servir des conclusions inverses. Ainsi notre exemple: "Não tínhamos X, (mas) agora temos", où: 1) - p suggère la conclusion $r$ : (le retard économique du pays); 2) - q suggére, en revanche, la conclusion non-r: (le développement économique de l'URSS - conclusion (thèse) d'ailleurs défendue par les orateurs.). Aussi, si nous nous référons à la théorie polyphonique de l'énonciation ${ }^{9}$, nous disons que l'énoncé ci-dessus met en scène deux énonciateurs différents (E1 et E2 - exprimant deux points de vue distincts), qui argumentent dans des sens opposés ( $p$ et $q$ ). $S$ 'assimilant à l'énonciateur qui asserte q (E2), le 
locuteur soviétique se distancie du premier énonciateur (E1), assimilé, lui, à un objecteur défendant un argument que disqualifierait q.

Il existe donc une convergence entre le schéma logique du discours propagandiste et l'enchaînement argumentatif des énoncés. En d'autres termes, la conclusion non-r va dans le sens de l'argumentation propagandiste, tissée, rappelons-le, autour de l'opposition passé vs présent. L'introduction du connecteur implicite "mas"(parfaitement adapté au co(n)texte, nous permet d'interpréter pertinemment $p$ et $q$, non pas en termes d'une simple opposition propositionnelle, mais en fonction de deux actes de langage accomplis par le locuteur: un acte "primitif", acte d'affirmation argumentative dans q, et un acte "dérivé", acte de concession ${ }^{10}$ dans p. Opposant deux énonciateurs (E1 et E2), à savoir, deux points de vue différents, p et q se caractérisent par une opposition argumentative.

Considérons les énoncés suivants du discours du personnage représentant le médecin soviétique constituant un nouveau type d'opposition entre arguments disjoints:

2a) - O Médico - “( . . .) Não temos mais as desigualdades e as infâmias produzidas pela herança burguesa. (...) Não temos mais adultério. (...)"(p. 198).

Dans ce deuxième cas de figure, la relation entre arguments a contrario s'établit, au premier abord, en fonction de l'opposition du posé et du présupposé. Si 2a) pose qu'actuellement les Soviétiques n'ont plus X (état de choses négatif), il présuppose qu'auparavant $X$ existait bel et bien. Le morphème negatif "não"étant en fait source de présupposition, et la présupposition, souligne $O$. Ducrot ${ }^{11}$, est partie intégrante du sens des énoncés. Or, au lieu d'affirmer explicitement l'existence de $\mathrm{X}$ dans le passé, le locuteur la nie donc au présent, tout en la présentant comme ayant été un fait irréfutable, c'est-à-dire une évidence incontestable. Le contenu du présupposé constitue donc une vérité acquise qui ne saurait être remise en question. Il convient, par ailleurs, d'observer le contenu même du posé dans le premier énoncé du segment $2 \mathrm{a}$ ). X, ou plus exactement, "as desigualdades e as infâmias", est explicité comme étant engendré "pela herança burguesa". Relevant incontestablement du passé, le présupposé d'existence de X ne saurait donc être réfuté.

On peut constater dans la séquence $2 a)$ que les deux énoncés sont en fait destinés à présenter les innombrables réalisations accomplies par les Soviétiques. Aussi ces énoncés véhiculent-ils des sous-entendus tels que: "Agimos de maneira eficaz."; "Somente o socialismo poderia combater 
$\mathrm{X}$."Tandis que la présupposition est partie intégrante du sens des énoncés, le sous-entendu concerne la façon dont ce sens doit être déchiffré par le destinataire ${ }^{12}$. Or, c'est bien l'idée d'action, transformation, efficacité relevant des sous-entendus, véhiculée par les énoncés, que le locuteurpersonnage entend donner par son énonciation, autrement dit, qu'il entend imposer à son destinataire, indépendamment des présupposés. L'insertion de 2a) dans son $\operatorname{co}(n)$ texte discursif original éclaircit par ailleurs le sens des énoncés, non seulement en confirmant les sous-entendus, mais en attestant l'utilisation des posés pour l'enchaînement du discours:2b) - O Médico - "( . . .) Não temos mais as desigualdades e as infâmias produzidas pela herança burguesa. Eliminamos com isso $90 \%$ das tragédias sociais. Não temos mais adultério. Não temos prostituição. Eliminamos as nevroses, os assassinatos, as depravações, (...) Matamos o monstro empolado do urbanismo. Liquidamos o desemprego."(p. 198)

L'utilisation d'énoncés à présupposés est destinée à enchaîner le discours apologétique du locuteur qui, dans son intégralité, présuppose l'existence antérieure d'un état de choses négatif, en renforçant au moyen des posés les clivages présent/passé, socialisme/capitalisme. Ayant pour but d'exposer les efforts accomplis par le régime soviétique, l'enchaînement discursif s'organise autour d'un certain nombre de verbes qui confirment, d'une part, le présupposé d'existence de X (comme par exemple, "eliminar", "suprimir", "matar", "liquidar"X) et, d'autre part, accentuent le rejet catégorique manifesté par les acteurs soviétiques contre $\mathrm{X}$ - faits de société - "produzidos pela herança burguesa", "apanágio da burguesia", comme le souligne le locuteur-personnage. Par ailleurs, l'aspect des verbes mentionnés - perfectif- ainsi que leur mode de procès - conclusifs (présentant dans les énoncés une action qui a atteint son terme) sont importants à relever, dans la mesure où, signalant l'achèvement du procès, ils servent à guider l'interprétation du destinataire dans le sens des sous-entendus. Aussi les arguments en faveur du présent sont-ils posés explicitement, non seulement pour servir de preuves à la prémisse initiale (Le développement en URSS est dû à l'implantation du système socialiste), en vue de défendre la thèse propagandiste (La construction socialiste représente le développement socioéconomique, la modernité), mais également pour enchaîner un discours qui se doit d'être précis et argumentativement transparent.

Le troisième type d'opposition entre arguments dichotomiques juxtaposés concerne une relation d'opposition sémantique sur le plan paradigmatique. Ainsi le discours attribué à Staline: - 3) - A Voz de Stalin - "Passar do cavalo camponês ao cavalo da indústria construtora de máquinas, eis o plano central do poder Soviético. Escutai a metáfora leninista. Passar 
de uma alimária à outra. Da alimária do campo, do cavalo que convém a um país arruinado de camponeses ao cavalo que o proletariado procura e deve procurar, o cavalo da indústria, o cavalo-vapor."(p. 187)

Dans le but de procéder à une description précise du projet soviétique, et, par là même, d'agir sur l'auditeur, l'orateur se sert donc de la "métaphore léniniste", tout en essayant d'expliciter les différences entre l'état de choses actuel et l'état de choses antérieur. Or, le passé est au présent ce que le retard économique est au développement industriel; autrement dit, $\mathrm{A}$ est à $\mathrm{B}$ comme $\mathrm{C}$ est à $\mathrm{D}$. Une telle homologie $(\mathrm{A}: \mathrm{B}: \mathrm{C}: \mathrm{D})$ constitue le raisonnement par analogie défini comme étant une similitude de relations entre termes qui appartiennent à des champs sémantiques différents. Si dans la séquence 3), la relation analogique est exprimée de façon implicite, c'est parce qu'il s'agit en fait d'une "analogie condensée"13; c'est-à-dire d'une fusion métaphorique d'un des éléments du phore avec un des éléments du thème. Tandis que le thème constitue l'ensemble des termes structurés (A et B) sur lequel porte la conclusion, le phore, c'est l'ensemble des termes (C et D) semblablement structurés qui servent à étayer le raisonnement. ${ }^{14}$

Le rôle argumentatif de la métaphore (opération langagière) est en fait intimement lié à celui de l'analogie (opération cognitive). Ceci dit, les expressions métaphoriques "cavalo camponês", "cavalo da indústria" résultent donc de l'accolement d'un terme du phore (le retard économique) et d'un terme du thème (le passé).

On peut dire, par ailleurs, que les expressions métaphoriques ci-dessus sont contraignantes et porteuses de jugement de valeur. En effet, les sèmes évaluatifs attribués au syntagme nominal "cavalo camponês", à savoir "archaïsme", "rusticité", correspondent à l'image que le destinataire (censé représenter le prolétariat urbain) se fait, dans son contexte spatio-temporel, d'un tel outil de travail. L'image dévalorisante de l'expression métaphorique "cavalo camponês"(ayant comme source l'analogie avec le passé) s'oppose, bien entendu, à l'image valorisante attribuée à "cavalo da indústria", véhiculant les sèmes "développement", "progrès", "productivité", que nul ne saurait contester. Le passage d'un "animal" à l'autre n'a été possible qu'avec la mise en oeuvre d'un nouveau système socio-économique. Or, la valeur argumentative des expressions métaphoriques est d'autant plus manifeste que le locuteur les réitère en les explicitant métaphoriquement. Qu' est-ce qu'un "cavalo camponês"? C'est "o cavalinho que convém a um país arruinado de componeses", et "o cavalo da indústria"(la polysémie du lexème "cavalo"permet un nouveau rapprochement analogique et une opposition sémantique avec la première expression métaphorique), c'est 
"o cavalo que o proletariado procura e deve procurar". Le développement des métaphores - s'expliquant par le souci catéchisant et, par là même, didactique d'un tel discours, permet alors au locuteur-orateur de poursuivre indirectement, ou plus exactement, au moyen d'un langage figuré, sa tâche persuasive.

\section{RÉFUTATION}

Faisant partie des "preuves", la réfutation constitue l'objection, le rejet des arguments "négatifs"(en faveur des thèses adverses), à savoir une contre-argumentation consistant à mettre en oeuvre différents moyens de mise en doute des positions opposées, ou simplement, à reprendre l'argument attribué à l'adversaire, dans le but, d'une part, de s'y opposer, et d'autre part, de renforcer la thèse défendue par le réfutateur. Une réfutation ne saurait se contenter d'attaquer et de nier l'argument de l'adversaire, sans pour autant justifier les raisons d'une telle négation, et expliciter ses propres arguments, afin de les rendre plausibles. Comme le souligne Aristote, "dans tous les cas où il y a eu réfutation, il est plus évident qu'il y a eu déduction; en effet, les contraires sont mieux connus quand ils sont mis en parallèle." ${ }^{15}$. Le double mouvement discursif de négation/correction, autrement dit, de réfutation/rectification et de réfutation/justification illustre les opérations linguistico-cognitives spécifiques à ce deuxième type de procédure logicoargumentative exploitée par le scripteur dans la mise en oeuvre des arguments.

Selon J. Moeschler ${ }^{16}$, la réfutation peut être caractérisée comme une fonction illocutoire réactive à une assertion, traduisant le désaccord de l'interlocuteur, désaccord qualifié d'évaluation négative. Il peut soit concerner le contenu propositionnel de l'acte d'assertion, soit concerner ses présupposés, ou le propre fait énonciatif. Dans le premier cas, le désaccord est de nature propositionnelle, dans le deuxième cas, de nature métalinguistique. Si d'une part, la réfutation (fonction illocutoire réactive) doit réaliser une infirmation, elle doit, d'autre part, contenir une argumentation; c'est-à-dire que la relation entre le composant négatif et le(s) composant(s) à fonction d'argument(s) de la réfutation est de nature argumentative. Les relations d'ordre argumentatif sont internes à la structure discursive de la réfutation. D'ailleurs, ce que caractérise le prédicat "réfuter"(pseudo-performatif), c'est la propriété de renvoyer à une argumentation. La condition d'argumentativité est une condition nécessaire pour les actes de réfutation. 
En effet, l'acte illocutoire de réfutation (ou fonction illocutoire réactive de réfutation) consiste en une énonciation d'un énoncé complexe formé d'un énoncé négatif (à négation polémique) et d'arguments. Or, l'intégration de la notion d'argumentativité dans le cadre d'une analyse fonctionnelle (pragmatique) des énoncés constitutifs d'une réfutation (l'acte de contenu $\mathrm{q}$ est une justification pour l'acte de contenu négatif non- $p$ ) permet à Moeschler de décrire la structure de la réfutation comme la composition d'un acte directeur (à fonction illocutoire d'assertion) et d'un acte subordonné (à fonction interactive ${ }^{17}$ de justification). L'existence d'une relation de subordination entre l'acte de contenu q et l'acte de contenu non- $p$ rend compte, en termes fonctionnels, de la différence, au plan argumentatif, entre les énoncés constitutifs de la réfutation. Ceci étant, la structure fonctionnelle d'une réfutation peut être représentée de la façon suivante: ASSERTION (non-p, JUSTIFICATION (q, ASSERTION $\left(\right.$ non-p))). ${ }^{18}$

Dans le discours de propagande de $O$ homem e o cavalo, le premier exemple de réfutation qui fera l'objet d'une analyse concerne la rectification, selon la terminologie proposée par J. Moeschler. Elle correspond aux énoncés négatifs dont l'élément sémantique (argument ou prédicat), c'est-à-dire le foyer ("focus") est spécifié par l'enchaînement. Autrement dit, ce type de réfutation porte sur un constituant de l'énoncé rejeté. Ainsi l'exemple suivant: "Tereza não é portuguesa, mas sim brasileira."

1) - A Voz de Eisenstein - "Eu vos apresento os documentos da transformação do mundo. A vitória encarniçada do proletariado na frente camponesa, na frente industrial. Nem bandeiras ao vento nem gritos nem canhões! Mas as cargas da cavalaria-vapor, na construção do socialismo! ( . . ) Nem o incêndio da revolta nem a grande luta revolucionária. Mas, depois da luta e da vitória, a vida quotidiana dos que trabalham e constroem um mundo melhor. (. . ) Nem amor da pátria nem Deus, nem a hipocrisia honesta. Mas os rebanhos que se organizam, os mapas da seleção de sementes, os diagramas do progresso. (. . . )"(p. 198)

La répétition et le parallélisme syntaxique se font autour des morphèmes marqueurs de réfutation, à savoir, les négations de type NEM...NEM et MAS. En effet, "mas"ne constitue pas dans l'exemple ci-dessus un connecteur d'argumentation, mais un connecteur de réfutation, correspondant à l'espagnol "sino". La structure syntactico-sémantique de 1) est: NEM $X, N E M ~ Z, N E M ~ Y$, MAS K, X, Z, Y correspondent à des arguments attribués à un énonciateur $\mathrm{E} 1{ }^{19}$, contestés et réfutés par le locuteur assimilé à $\mathrm{E} 2$, tandis que $\mathrm{K}$ correspond à l'argument neuf, positif, introduit par 
le locuteur-orateur, c'est-à-dire à une assertion vraie qui vient à son tour rectifier toute la phrase. Or, les arguments attribués à $\mathrm{E} 1$, et rejetés par le locuteur, sont, dans chaque énoncé, au nombre de trois (ou deux), ce qui veut dire qu'à chaque fois, il y a une triple (ou double) négation, ou plus exactement, réfutation, suivie d'une rectification introduite par "mas". Or, le développement de $\mathrm{K}$ ("conjoint antonymique"de $\mathrm{X}, \mathrm{Z}, \mathrm{Y}$ ) atteste l'importance accordée à cet argument, par le locuteur, qui se doit d'être explicite pour aboutir à disqualifier entièrement ceux de l'adversaire, c'està-dire $\mathrm{X}, \mathrm{Z}, \mathrm{Y}$ (attribués a $\mathrm{E} 2$ ), et à répondre à la question: qu'est-ce que “a vitória encarniçada do proletariado na frente camponesa ( . . .)?"Les réponses correspondent aux arguments $\mathrm{K}$, c'est-à-dire:

- K 1 - "as cargas de cavalaria-vapor, na construção do socialismo!";

- K 2 - "a vida quotidiana dos que trabalham e constroem um mundo melhor";

- K 3 - "os rebanhos que se organizam ( . . ), diagramas do progresso."

Disparates, au premier abord, les assertions de l'orateur (constituant les rectifications) sont cependant des arguments pertinents, dans la mesure où ils sont énoncés pour défendre la conclusion globale (thèse valorisante), à savoir: "la construction socialiste représente le développement socioéconomique, la modernité."

Soit la séquence suivante:

2) - São Pedro - "Eu desejava somente saber quais os resultados dessa transformação, desse milagre...

- O Médico - Não foi milagre. Nada é misterioso na aplicação prática de ciência social. ( . . . )"(p. 198)

Il s'agit donc d'une réfutation de caractère dialogal, ou plus exactement d'un échange réfutatif, dans la mesure où l'acte de réfutation fait suite au discours de l'adversaire, c'est-à-dire aux deux actes d'énonciation formulés par 1'interlocuteur, le personnage São Pedro: une requête (demande d'information) et une assertion sous-jacente ("essa transformação (é um) milagre.") - interprétée comme un acte de prédication - cible de la réfutation. L'acte de réfutation (réactif) a donc pour origine une assertion préalable (initiative) et se réalise à l'intérieur d'une interaction polémique (un échange) donnant lieu à la longue intervention d'explicitation (discours monologal développé par le locuteur propagandiste), qui, elle, constitue la réponse 
à la demande d'information formulée au départ par l'interlocuteur. Constituée d'un énoncé négatif et d'un énoncé à fonction de justification (l'argument), la réfutation en 2) équivaut à ce que J. Moeschler appelle la réfutation propositionnelle. La présence de l'acte de justification s'explique d'ailleurs par la condition d'argumentativité imposée aux actes assertifs.

\section{ILLUSTRATION}

Troisième type de procédure logico-argumentative mise en oeuvre dans le discours, l'illustration (à côté de l'opposition et de la réfutation) constitue, en fait, l'étape décisive de l'argumentation propagandiste (monologal) où toute une série de "faits", c'est-à-dire de données susceptibles d'être observées, sont présentés à titre de preuve.

Considérée par Ch. Perelman et L. Olbrechts-Tyteca comme un argument de type particulier, ou plus exactement, l'un des trois types d'arguments fondés sur le "cas particulier", à côté de l'exemple et du modèle ${ }^{20}$, l'illustration vise à renforcer l'assentiment à une règle déjà formulée ou implicitement admise; sa fonction est, en l'occurrence, de corroborer une règle déjà existante, en exhibant son intérêt par la variété de ses applications. Aussi l'illustration vise-t-elle à accroître la présence, en concrétisant à l'aide d'un "cas particulier"une règle abstraite. Il convient de rappeler que la "règle"au sens où les deux auteurs en question en traitent, "c'est tout énoncé général par rapport à ce qui en est une application"21. Ceci étant, la qualification attribuée à une personne peut être considérée comme une règle dont les comportements fourniraient des illustrations. Bref, l'illustration vise à étayer une régularité déjà établie.

En fournissant des cas particuliers, et ce, pour éclairer l'énoncé général, c'est-à-dire la règle, l'illustration est souvent présentée dans une argumentation sous forme d'une énumération de cas, pouvant se produire dans un registre lapidaire, ou au contraire, être développés à l'aide de détails descriptifs, afin de mieux transmettre les précisions visées. Le discours radiophonique de l'orateur propagandiste représentant Eisenstein explicite le premier cas de figure:

- A Voz de Eisenstein - “( . . ) Na nossa gota d'água se reflete o horizonte da nova era social. Estações experimentais. Fazendas modelos. Laboratórios, escolas. O operário estudante, o camponês estudante. A reprodução consciente e selecionada das espécies animais. $O$ fim da magia. O trator. ( . . . )"(p. 189) 
En effet, les énumérations lexicales constituent une illustration de la règle admise, c'est-à-dire de la conclusion (Le socialisme représente la "nouvelle ère sociale") formulée partiellement dans le premier énoncé. Les arguments fournis par l'orateur pour accroître l'adhésion à la thèse défendue se présentent sous la forme de lexèmes et de syntagmes comportant tous un trait "valorisant", spécifique au concept axiologique de "développement socio-économique"- "objet d'accord"relevant du "préférable", autrement dit, valeur censée être unanimement admise par 1'auditoire. Aussi la valorisation des faits matériels ("cas particuliers") se fait-elle dans le discours, par le biais de termes lexicaux exprimant l'idée de "rationalité", "planification", "instruction"afférente aux nouvelles pratiques socio-économiques instaurées en URSS, grâce aux radicales transformations des rapports de production.

Il convient, par ailleurs, de souligner que les arguments présentés par l'orateur reposent sur le lieu axiologique qui prône la supériorité de la "qualité", à travers la valorisation de l'unique. La valeur de l'unique, comme l'affirme Perelman, peut s'exprimer par son opposition au commun, au banal, au vulgaire constituant la forme dépréciative du multiple ${ }^{22}$. Or, la spécificité du système soviétique ("lieu de l'unique") est présentée pour s'opposer à la norme, autrement dit, à l'absence d'originalité et de hardiesse caractéristique des politiques capitalistes. Ceci étant, le caractère exemplaire des réalisations accomplies par les socialistes - servant d'illustration de la thèse défendue - constitue l'un des pivots de l'argumentation propagandiste.

Soit le deuxième exemple d'illustration:

- O Médico - "( . . . ) Possuímos 2000 maternidades gratuitas. Temos 10000 creches. Colocadas ao lado das fábricas, dos laboratórios, das universidades. ( . . . ) A construção do socialismo apresenta um considerável melhoramento moral, educacional e sobretudo material das massas operárias e colcozianas. A mortalidade baixou a $1 / 3$ da cifra antiga. ( . . .) O número de postos médicos, de creches e de leitos aumenta ano a ano. A melhoria sanitária é notável nas empresas socialistas gigantes e nas regiões nacionais. Onde havia 60 leitos existem agora $2525,(\ldots)$ temos 85000 médicos servindo o povo. Antigamente havia só 19.000 a soldo das classes ricas. ( . . . ) O número de leitos nas creches de verão dos colcozes atingiu a dois milhões. Vai ser aumentado de $34 \%$ o número de médicos para crianças e adolescentes. As cozinhas láteas coletivas aumentaram duas vezes e meia."(pp. 198, 199)

Pour corroborer la thèse (une fois encore explicitée dans le discours: "A construção do socialismo apresenta um considerável melhoramento moral, 
educacional e sobretudo material das massas operárias ( . . .)") - constituant la règle admise - l'illustration dans 2) fait appel à un nombre significatif de données chiffrées (l'emploi d'adjectifs numéraux y est abondant) qui sont avancées à l'appui d'assertions de type informatif visant à modifier le bagage cognitif de l'auditoire, et, en l'occurrence, à le persuader du bienfondé de l'argumentation.

La mise en parallèle de la dimension temporelle (passé vs présent) - par le biais d'une démarche rationnelle (chiffres à l'appui) éclaircissant par des cas particuliers la règle admise - a donc pour corollaire de renforcer l'adhésion à cette règle. Ceci étant, l'énumération des faits relevant du temps "présent" est indissociable de leur évaluation. La confrontation des deux périodes vise, bien entendu, à évaluer l'une au détriment de l'autre.

Contrairement à l'exemple précédent 1), le discours en 2) repose sur le "lieu de la quantité" axé sur le postulat selon lequel une chose vaut mieux qu'une autre, pour des raisons quantitatives. Les arguments fondés sur la supériorité numérique constituent, par la valorisation directe, un "lieu axiologique"23.

En faisant appel à des données numériques pour clore son allocution, l'orateur-médecin se sert de preuves dont la force argumentative confère au discours un poids persuasif supplémentaire. Si de tels procédés prétendent à une certaine validité dans l'exposition des faits (éléments cognitifs), l'efficacité de l'illustration est également fonction de leur évaluation inséparable, d'une part, de la mise en jeu des arguments disjoints (passé vs présent), et, d'autre part, de l'acte même d'argumenter faisant toujours valoir une idée, un point de vue, un jugement.

\section{EXPLICITATION DE LA THÈSE}

Il convient de rappeler que la conclusion explicite soutenue par les orateurs se trouve, à plusieurs reprises, réitérée dans le discours des propagandistes. L'argumentation débute d'ailleurs, par la formulation de la thèse défendue. En effet, la proposition initiale de l'allocution du premier orateur-personnage comporte la définition de "socialisme" formulée par Lénine.

- A Voz de Stalin - "O Socialismo é o poder dos Soviets mais a eletrificação. Eis o testamento de Lênin. ( . . .) Do século da madeira passamos ao século do motor e do aço. A economia agrícola repousa agora sobre a base técnica da grande produção moderna."(p. 186) 
Si des données polémiques (coexistence d'éléments constatatifs et de jugements de valeurs, le renforcement d'un point de vue) déterminent souvent une définition, on ne saurait la spécifier, sans se référer à sa fonction persuasive: son rôle dans la persuasion consistant à faire valoir des éléments qui sont les principes d'une argumentation ${ }^{24}$. Aussi l'énonciation de la thèse (citation de Lénine) au début de l'argumentation propagandiste viset-elle d'emblée à présenter la position de l'orateur, en affichant ses premières assertions et jugements, et à exposer préalablement la finalité du discours, à savoir, la défense des vertus du socialisme, la réussite du régime soviétique. Ce faisant, l'orateur soviétique manipule des a priori, en insistant sur les notions de "croissance économique", "pouvoir populaire"- supposées être acceptées par l'auditoire - afin de valoriser les significations qui leur sont associées, telles le nouveau, la technicité, les réalisations. Or, la thèse défendue par les orateurs s'appuie sur ces concepts-valeurs de base qui sous-tendent l'argumentation propagandiste dans son intégralité.

Pour mieux expliciter ce qu'est la "construction socialiste"et, en l'occurrence, accroître l'adhésion de l'auditoire, la formulation de la thèse (insérée dans le discours parmi les différents arguments avancés par les orateurs) y revient à maintes reprises, le plus souvent de manière fragmentaire. En d'autres termes, le rappel de la thèse se fait partiellement, et ce, à chaque fois que les concepts axiologiques de nouveau ${ }^{25}$, réalisations, technicité émergent à la surface du discours, dans le but de qualifier la "construction soviétique". Ainsi les exemples:

2) - A Voz de Stalin - "Passar do cavalo camponês ao cavalo da indústria construtora de máquinas ( . . . )"(p. 187)

3) - A Voz de Stalin - "( . . .) Sobre as fábricas entregues ao trabalhador surgiu o entusiasmo da nova sociedade. É o pathos da construção!'(p. 187)

4) - A Voz de Eisenstein - "( . . . ) depois da luta e da vitória, a vida quotidiana dos que trabalham e constroem um mundo melhor. ( . . . )"(p. 189)

5) - A Voz de Eisenstein - “( . . ) Edificamos. Na nossa gota de água se reflete o horizonte infinito da nova era social. ( . . . )"(p. 189)

6) - A Voz de Eisenstein - “( . . ) Inaugura-se por toda a terra coletivizada a época do vapor e da eletricidade. ( . . . ) (p. 189)

7) - A Voz de Eisenstein - “( . . ) Fazemos a Industrialização". (p. 189)

La formulation finale de la thèse, sous forme de synthèse, met l'accent sur les profondes transfomations survenues au sein de la société soviétique, autrement dit, sur les nouvelles conditions de vie du peuple soviétique 
(valorisation du travailleur, augmentation du pouvoir d'achat; accès à l'éducation) correspondant aux valeurs auxquelles est censé adhérer l'auditoire, à savoir, les "trabalhadores do mundo".

8) - O Médico - "( . . ) A construção do socialismo apresenta um considerável melhoramento moral, educacional e sobretudo material das massas operárias e colcozianas. ( . . . )"(p. 199)

\section{NOTES}

${ }^{1}$ Dans la thèse de Doctorat d'Etat, Le fonctionnement du discours d'agitprop dans $\boldsymbol{O}$ homem e o Cavalo d'Oswald de Andrade (Université de Franche-Comté, 1991), j'ai défendu l'hypothèse de la présence du discours d'agit-prop dans la pièce en question; le discours politique militant s'y réalisant par le biais du discours d'agit-prop.

${ }^{2}$ Oswald de Andrade, Obras Completas VIII, Teatro, Rio, Civilização Brasileira, 1978, p. 183.

3" (. . . ) un trait sémantique présent dans l'un des termes est absent de l'autre." (Marc Angenot, La parole pamphlétaire (Typologie des discours modernes), Paris, Payot, 1982, p. 115.)

${ }^{4} \mathrm{Ce}$ "lieu" dit que si un accident est à une chose, la négation (ou le contraire) de la chose est au contraire de l'accident. (M. Angenor, ibid., p. 384)

${ }^{5}$ Dans Dire et ne pas dire, $O$. Ducrot distingue deux types de négation, selon leur fonction dans les actes d'énonciation. Alors que la négation "descriptive"sert à représenter un état de choses (ex: "il n'y a pas un nuage au ciel"), la négation "polémique"sert, au contraire, à contrer une opinion inverse, c'est-à-dire à s'opposer à une affirmation antérieure (ex. "Ce mur n'est pas blanc").

${ }^{6} \vdash$ est le marqueur de force illocutoire d'assertion et est le symbole logique de négation (Jacques Moeschler, Dire et contredire (Pragmatique de la négation et acte de réfutation dans la conversation), Berne, Lang, 1982, p. 30).

7“Deux mais en français?”, Lingua, 43, 1977.

${ }^{8} \mathrm{~J}$. Moeschler, Argumentation et conversation (Eléments pour une analyse pragmatique du discours), Paris, Hatier-Credif, 1985. 
${ }^{9} \mathrm{Cf}$. O. Ducrot, Le dire et le dit, Paris, Minuit, 1984.

10“" ( . . ) il consiste à faire entendre un énonciateur argumentant dans un sens opposé au sien, énonciateur dont on se distancie (tout en lui donnant, dans le cas au moins des concessions introduites par certes, une certaine forme d'accord)." Ibid., p. 230.

${ }^{11}$ Ibid., p. 44.

${ }^{12}$ Ibid., p. 44.

${ }^{13}$ Chaïm Perelman et Lucie Olbrechts-Tyteca, Traité de l'argumentation (La Nouvelle Rhétorique), Editions de l'Université de Bruxelles, 5e édition, 1988 , p. 535.

${ }^{14} \mathrm{~L}$. Olbrechts-Tyteca, Le comique du discours, Edit. de l'Université de Bruxelles, 1977, p. 288.

${ }^{15}$ Rhétorique, Livre III, 17, 1418 a.

${ }^{16} \mathrm{Cf}$. Dire et contredire, op. cit.

17" ( . . . ) le caractère intrinsèquement relationnel et non illocutoire de la justification, nous permet de qualifier cet acte d'interactif." (Ibid, p. 131).

${ }^{18}$ Ibid., p. 132.

${ }^{19}$ Dans le cadre de la conception polyphonique de l'énonciation, la négation "polémique"est explicitée para $\mathrm{O}$. Ducrot (Le dire et le dit), comme relevant de deux points de vue opposés. En effet, lors d'une énonciation d'un énoncé déclaratif négatif, le locuteur $L$ fait apparaître deux attitudes antagonistes: l'une positive attribuée à un énonciateur $\mathrm{E} 1 \mathrm{mis}$ en scène dans le discours, et l'autre, correspondant à un refus de la première, attribuée à $\mathrm{E} 2$, assimilé au locuteur.

${ }^{20}$ Traité de l'argumentation, op. cit., chapitre III.

${ }^{21} I$ bid., p. 486.

${ }^{22}$ Ibid., p. 121.

${ }^{23} \mathrm{M}$. Angenot, La parole pamphlétaire, op. cit., pp. 131, 132.

${ }^{24}$ Ibid., p. 138. 
25“Dans la publicité et dans la propagande, la stratégie persuasive s'appuie inévitablement sur la révélation du nouveau, qu'il soit réel ou apparent. Mais l'apparent est ici réalité." (Denis Huisman, Le dire et le faire (Essais sur la communication efficace), Paris, SEDES, 1983, p. 132).

Profa. Dra. Heliane Kohler-Rodrigues

Maître de conférences - Section d'Espagnol/Portugais

Faculté des Lettres et Sciences Humaines de l'Université de Franche-Comté (Besançon) 